\section{Conceptos básicos de las inmunodeficiencias primarias}

Claudia Hernández-Martínez, ${ }^{1}$ Francisco Espinosa-Rosales, ${ }^{1}$ Sara Elva Espinosa-Padilla, ${ }^{1}$ Ana Rosa Hernández-Martínez, ${ }^{1}$ Lizbeth Blancas-Galicia ${ }^{1}$

\section{Resumen}

Las inmunodeficiencias primarias son un grupo heterogéneo de trastornos hereditarios ocasionados por defectos del desarrollo o función del sistema inmunológico, la mayoría se manifiestan a edad temprana por infecciones, datos de malignidad o por disregulación en la respuesta inmune, ya sea autoinflamación, autoinmunidad o alergia. Debido a que las inmunodeficiencias primarias son trastornos genéticos, la mayoría se heredan de forma autosómica recesiva. Las inmunodeficiencias primarias son más prevalentes en el sexo masculino y en edad pediátrica; las inmunodeficiencias de anticuerpos son las inmunodeficiencias primarias con más prevalente de la edad adulta. Dentro de las manifestaciones clínicas más comunes están la infección de vías respiratorias seguida de infecciones de la piel, abscesos, candidiasis, diarrea, BCGosis, etc. El abordaje inicial debe contar con una biometría hemática completa y cuantificación de inmunoglobulinas. El retraso del diagnóstico se debe principalmente a que las infecciones recurrentes pueden ser aceptadas como variaciones de la normalidad o a una percepción errónea de que su presentación es exclusiva de la infancia. El reconocimiento temprano por cualquier médico de primer contacto es importante para el tratamiento oportuno y el mejor prónostico.

PALABRAS CLAVE: Inmunodeficiencias primarias, enfermedades con inmunodeficiencias, infecciones recurrentes, tratamiento con inmunoglobulina, profilaxis.

Rev Alerg Méx 2016 Apr-Jun;63(2):180-189.

\section{Basics of primary immunodeficiencies}

Claudia Hernández-Martínez, ${ }^{1}$ Francisco Espinosa-Rosales, ${ }^{1}$ Sara Elva Espinosa-Padilla, ${ }^{1}$ Ana Rosa Hernández-Martínez, ${ }^{1}$ Lizbeth Blancas-Galicia ${ }^{1}$

\begin{abstract}
Primary immunodeficiencies (PID) are a heterogeneous group of inherited disorders, the etiology are the defects in the development or function of the immune system. The principal PID manifestations are the infections in early age, malignancy and diseases of immune dysregulation as autoimmunity and allergy. PIDs are genetics disorders and most of them are inherited as autosomal recessive, also this group of diseases is more prevalent in males and in childhood. The antibody immunodeficiency is the PID more common in adults. The more frequent disorders are the infections in the respiratory tract, abscesses, candidiasis, diarrhea, BCGosis etc. Initial approach included a complete blood count and quantification of immunoglobulins. The
\end{abstract}

${ }^{1}$ Unidad de Investigación en inmunodeficiencias, Instituto Nacional de Pediatría, Secretaría de Salud Distrito Federal, México

Recibido: 6 de noviembre 2015

Aceptado: 21 de enero 2016

Correspondencia

Dra. Lizbeth Blancas-Galicia

Unidad de Investigación en Inmunodeficiencias

Torre de Investigación, piso 9,

04530 Ciudad de México

blancas.lizbeth@gmail.com

Este artículo debe citarse como

Hernández-Martínez C, Espinosa-Rosales F, EspinosaPadilla SE, Hernández-Martínez AR, Blancas-Galicia L. Conceptos básicos de las inmunodeficiencias primarias. Rev Alerg Méx. 2016 abr-jun;63(2):180-189. 
delay in diagnosis could be explained due to a perception that the recurrent infections are normal process or think that they are exclusively of childhood. The early diagnosis of PID by primary care physicians is important to opportune treatment and better prognosis.

KEYWORDS: primary immunodeficiency, immunodeficiency diseases, recurrent infections, immunoglobulin therapy, prophylaxis.

\author{
Correspondence \\ Dra. Lizbeth Blancas-Galicia \\ Unidad de Investigación en Inmunodefi- \\ ciencias \\ Torre de Investigación, piso 9, \\ 04530 Ciudad de México \\ blancas.lizbeth@gmail.com
}

\section{INMUNODEFICIENCIAS PRIMARIAS}

La función primordial del sistema inmune es la diferenciación entre los antígenos propios y los no propios (por ejemplo, agentes infecciosos); cuando existe una alteración en él, las manifestaciones clínicas más frecuentes son las infecciones, así como la autoinflamación, la autoimunidad, las alergias y las neoplasias. ${ }^{1}$

En el proceso de protección contra la propagación de agentes infecciosos en el organismo, solo algunos individuos experimentan infecciones que se salen de control. El trastorno cuya causa es un defecto genético en uno o más componentes del sistema inmune recibe el nombre de inmunodeficiencia primaria (IDP), originada por la alteración de una o más proteínas del sistema inmune celular o humoral, lo que explica la diversidad o heterogeneidad en la susceptibilidad particular a diferentes agentes infecciosos. Es importante hacer énfasis en que las inmunodeficiencias primarias (IDP) no están relacionadas con el virus de la inmunodeficiencia humana (VIH) ni son contagiosas. ${ }^{2}$

\section{EPIDEMIOLOGÍA}

En 1952, O. Bruton describió la primera IDP, la agammaglobulinemia ligada al cromosoma X; más tarde se identificó que el responsable es el gen mutado de BTK. Los avances en biología molecular han favorecido el incremento explosivo en la caracterización de nuevas inmunodeficiencias primarias. La prevalencia de las IDP en los países desarrollados es de 1 entre 200000 nacidos vivos. En Francia, la incidencia es de 1 entre 400 000, de modo que se estima que cada año nacen de 150 a 200 niños con IDP.

Tanto la distribución como la prevalencia de las IDP varían entre los distintos grupos humanos debido a las características genéticas y al acceso a los recursos diagnósticos.

En cuanto al sexo, las IDP son más frecuentes en hombres debido al patrón de herencia ligado al cromosoma X. En cuanto a la clasificación, son más frecuentes las IDP que afectan al sistema humoral (Cuadro 1). En México, los datos epidemiológicos de las IDP se registran en la página electrónica de LASID (Latinoamerican Society for Inmunodeficiency). ${ }^{3-6}$

\section{CLASIFICACIÓN DE LAS IDP}

Se han propuesto diversas clasificaciones de las IDP con base en criterios como la edad de inicio, el tipo de susceptibilidad a los agentes infecciosos y la localización de la infección. La clasificación más reciente, de 2015, fue propuesta por expertos de la Unión Internacional de Sociedades de Inmunología" (IUIS, International Union of Immunological Societies). En ella se describen nueve grupos: inmunodeficiencias que afectan la inmunidad celular y humoral, inmu- 
Cuadro 1. Clasificación de las inmunodeficiencias primarias

Deficiencia de linfocitos B (humoral)

Deficiencia de linfocitos $T$ (celular)

Deficiencia de linfocitos B y T (combinada)

Defectos de fagocitosis

Defectos de complemento

Asociadas a un fenotipo característico

Otras

nodeficiencias combinadas con características específicas o relacionadas con un síndrome, deficiencias predominantemente de anticuerpos, enfermedades de disregulación inmune, defectos congénitos en el número o función de fagocitos, defectos en la inmunidad intrínseca e innata, trastornos autoinflamatorias, deficiencias del complemento y fenocopias de IDP.

Existe otra clasificación útil para orientar acerca del tipo de estudios que pueden solicitarse en caso de sospecha diagnóstica (ver más adelante la sección de diagnóstico). ${ }^{17,8}$

\section{GENÉTICA}

Las IDP son enfermedades con una etiología genética o hereditaria: un gen sufre una mutación que se traduce en la síntesis anormal de una proteína, lo que da lugar a una susceptibilidad anormal de un agente infeccioso, con un patrón de herencia determinado. Una misma mutación en un mismo gen específico puede generar diferentes expresiones fenotípicas. A la fecha se has descrito más de 300 genes responsables de IDP. ${ }^{7}$

Por otro lado, existen individuos con clara susceptibilidad a infecciones en quienes no se ha detectado un gen responsable; de su estudio surgirán las nuevas descripciones de las IDP. En los últimos años, el descubrimiento de los genes responsables de las IDP ha permitido entender mejor el desarrollo y la función del sistema inmune. ${ }^{3}$
Los dos tipos de trasmisión hereditaria más frecuentes son el ligado al cromosoma $X$ (antecedentes familiares de varones fallecidos durante la infancia temprana) y el autosómico recesivo (la consanguinidad entre los padres incrementa la posibilidad); otro es el autosómico dominante. Algunas familias no tienen un tipo de herencia definido aun cuando hay más de un integrante afectado y con mayor susceptibilidad a las infecciones (antecedente de muerte en la infancia de individuos de uno u otro sexo). ${ }^{9}$ El antecedente familiar negativo no excluye la posibilidad de una IDP debido a que pueden darse algunas de las siguientes situaciones:

- La madre es portadora de una enfermedad ligada al cromosoma $X$ y ella no lo sabe.

- Ambos padres son portadores de una enfermedad autosómica recesiva.

- La anomalía genética es de novo, por una mutación durante el proceso de fertilización del espermatozoide al ovocito.

\section{EDAD DE PRESENTACIÓN}

Las IDP pueden evidenciarse a cualquier edad, sin embargo, con frecuencia se manifiestan durante la infancia ( $55 \%$ del total de casos). La inmunodeficiencia combinada severa afecta la respuesta inmune humoral y celular y se presenta desde el nacimiento; otras inmunodeficiencias pueden manifestarse durante la edad adulta, principalmente las humorales, como la inmunodeficiencia común variable $(87 \%$ de todos los casos son adultos). Recientemente se han descrito pacientes adultos con infecciones por micobacterias atípicas y mutaciones en los genes IRF8 y GATA2. ${ }^{10-13}$

\section{INFECCIONES EN INMUNODEFIENCIAS PRIMARIAS}

Las IDP se manifiestan principalmente con infecciones, cuyas características pueden hacer sospechar el diagnóstico: 
1. Recurrencia. No hay un evento aislado infeccioso, sino que las infecciones son recurrentes y su cronicidad puede llevar a daño permanente de un órgano. El diagnóstico diferencial de la neumonía recurrente relacionada con bronquiectasia incluye la inmunodeficiencia humoral. De igual forma, la recurrencia de otitis o sinusitis debe hacer pensar en inmunodeficiencia de antígenos polisacáridos específicos. ${ }^{14}$

2. Severidad. Algunos pacientes con IDP comienzan sus manifestaciones con infecciones banales que posteriormente evolucionan a eventos severos por los que requieren hospitalización, por ejemplo, por sepsis, meningitis o abscesos profundos (en hígado o anal). ${ }^{8}$

3. Agentes etiológicos. Las IDP pueden ser causadas por agentes etiológicos oportunistas (micobacterias, Nocardia, Aspergillus o Pneumocystis, entre otros). El tipo de susceptibilidad infecciosa depende de la IDP, de ahí la importancia de los aislamientos, por ejemplo: ${ }^{1}$

- A bacterias y enterovirus, inmunodeficiencias humorales.

- A bacterias y hongos, enfermedad granulomatosa crónica.

- A virus, hongos y bacterias, inmunodeficiencia combinada severa.

- A micobacterias o salmonella, inmunodeficiencia por defecto del receptor IL12RB1.

Si bien desde hace tiempo se registró que un individuo padecía infecciones recurrentes por un solo agente infeccioso, apenas hace dos décadas se identificó que el trastorno de una sola molécula del sistema inmune es capaz de generar susceptibilidad a un solo germen, por ejemplo, la alteración de TLR3 produce sensibilidad a encefalitis herpética; de STAT1, a candidiasis mucocutánea; de IL12RB1, a salmonelosis. Ca- sanova comparó las características de las IDP en 1952 y en 1996 (Cuadro 2). ${ }^{15}$

4. Cronicidad. La infección no mejora con los tratamientos ordinarios (por ejemplo, la candidiasis que tiene su base en el defecto de la molécula CARD 9). ${ }^{16}$

5. Respuesta a agentes antimicrobianos. No hay mejoría satisfactoria con la administración de antibióticos, y hay recaída cuando éstos se suspenden por haberse completado el esquema o se tienen que emplear esquemas terapéuticos más agresivos, largos o combinados (antibióticos más antimicóticos o antivirales). ${ }^{3}$

6. Reacciones adversas a vacunas. Las vacunas de agentes vivos atenuados pueden causar infección en pacientes con IDP. La infección por la vacuna BCG se observa en enfermedad granulomatosa crónica, inmunodeficiencia combinada severa y en pacientes con defectos del eje IL12/IFN $\gamma$. La infección por la vacuna de Sabin se observa en pacientes con agammaglobulinemia. El antecedente de reacción adversa por administración de agentes vivos debe ser un signo de alerta. ${ }^{17}$

La Fundación Jeffrey Modell ha descrito y difundo 10 signos de alarma que pueden indicar la existencia de una IDP, sin embargo, con el tiempo se ha demostrado que pueden pasarse

Cuadro 2. Evolución del concepto de las infecciones en las inmunodeficiencias IDP en las primeras descripciones, según Casanova

\begin{tabular}{cc}
\hline 1952 & 1996 \\
Múltiples & Única \\
Recurrente & Única (aguda o crónica) \\
En la Infancia & A cualquier edad \\
Gérmenes oportunistas & No necesariamente \\
Raras & Raras o comunes \\
Familiares & Esporádicas \\
\hline
\end{tabular}


por alto ciertos casos. El Centro de Referencia de Déficits Inmunitarios Hereditarios (CEREDIH, Centre de Référence Déficits Immunitaires Héréditaires), uno de los más calificados en Europa, ha sugerido signos de alarma para IDP tanto en niños como en adultos (Cuadro 3). ${ }^{18}$

\section{OTRAS CARACTERÍSTICAS CLÍNICAS NO INFECCIOSAS}

Patrón familiar. Antecedente de muertes en la infancia sin una etiología determinada. ${ }^{15}$

Peso y talla. En la infancia, la cronicidad de las infecciones relacionadas con IDP tiene repercusión en el peso y la talla, sin embargo, la talla y el peso normales no descartan una IDP. Algunos adultos con inmunodeficiencia común variable cursan con granulomas en intestino y peso bajo. ${ }^{1}$

Tejido linfoide. La ausencia de amígdalas y ganglios se observa en las agammaglobulinemias (ausencia de inmunoglobulinas séricas), así como la ausencia de timo en la inmunodeficiencia combinada severa o síndrome de DiGeorge. ${ }^{14}$

Fenotipos clínicos característicos. En general, las IDP se presentan con mayor susceptibilidad a infecciones sin ninguna otra manifestación, pero en ocasiones tienen un fenotipo clínico característico como implantación baja de orejas, micrognatia, hipertelorismo, úvula bífida y defectos cardiacos en el síndrome de Di George; facies tosca en el adulto con síndrome de hiperlgE (patrón autosómico dominante); petequias y eccema en la enfermedad de Wiskott-Aldrich; cabellos plateados en los síndromes de Griscelli y Chediak-Higashi; así como telangientasias oculares en el síndrome de ataxia telangiectasia. ${ }^{14}$

Autoimunidad. En general, los pacientes con IDP tienen mayor riesgo (respecto a la población general) de presentar eventos autoinmunes como
Cuadro 3. Signos de alarma sugeridos por el Centre de Référence Déficits Immunitaires Héréditaires

Signos clínicos que deben hacer pensar en una IDP en el adulto

1. Más de 2 otitis por año.

2. Más de 2 sinusitis aguas o crónicas en un año.

3. Más de 2 meses de tratamiento con antibiótico o necesidad de tratamiento antibiótico por vía intravenosa.

4. 2 neumopatías al año.

5. Diarrea crónica con pérdida de peso.

6. Episodios de fiebre alta, inexplicable.

7. Micosis mucocutánea persistente.

8. Dos infecciones graves en un año.

9. Un caso de inmunodeficiencia ya conocido en la familia.

10. Manifestaciones autoinmunes o granulomas.

11. Infecciones virales de repetición o crónicas (herpes, verrugas, aftas, condilomas, infecciones genitales en la mujer).

12. dilatación de bronquios y/o bronquitis de repetición sin una causa conocida.

Signos clínicos que deben hacer pensar en una IDP en el niño

1. Infecciones recurrentes de vías respiratorias altas y bajas.

o Más de 8 otitis por año en menores de 4 años.

o Más de 4 otitis por año en niños de más de 4 años de edad.

2. Más de 2 de neumonías o más de 2 sinusitis anualmente.

3. Infecciones severas con gérmenes de tipo neumococo, Haemophilus o Neisseria. Un solo evento de sepsis o meningitis debe ser motivo para sospechar la existencia de IDP.

4. Infecciones recurrentes con un mismo patógeno.

5. Infecciones habituales con evolución inhabitual (diarrea infecciosa persistente, algodoncillo o candidiasis cutánea recidivante).

6. Interrupción del incremento en el peso y la talla o diarrea persistente.

7. Antecedentes heredofamiliares de IDP o signos clínicos que la sugieran.

anemia hemolítica, plaquetopenia, vitíligo, lupus eritematoso sistémico o anemia perniciosa. ${ }^{3}$

Neoplasias. Al igual que la autoinmunidad, los pacientes con IDP tienen mayor riesgo de padecer neoplasias, principalmente linfoma. ${ }^{19}$ 


\section{INMUNODEFICIENCIAS PRIMARIAS ¿SIN INFECCIONES?.}

En la clasificación de IDP propuesta en 2015 por la Unión Internacional de Sociedades de Inmunología se reconoce que éstas pueden presentarse sin infecciones, tan solo con eventos autoinflamatorios como fiebre, artritis, rash, enfermedad inflamatoria intestinal o angioedema. ${ }^{7}$

\section{INMUNODEFICIENCIAS PRIMARIAS EN EL ADULTO}

Las inmunodeficiencias humorales son las más frecuentes en el adulto. En este grupo de edad, además de las infecciones, las manifestaciones autoinmunes (anemia hemolítica autoinmune, trombocitopenia, vitíligo, tiroiditis, lupus, anemia perniciosa), la esplenomegalia o las adenopatías con hiperplasia folicular, timoma, diarrea crónica, granulomas, poliartritis atípica pueden sugerir una IDP (Cuadro 3). ${ }^{4}$

\section{DIAGNÓSTICO}

La premisa para diagnosticar una IDP es reconocer que si bien se catalogan como enfermedades raras, son una realidad médica y no un mito. En el diagnóstico de presunción deben descartarse las inmunodeficiencias secundarias a: $:^{1,3,8}$

- Causas mecánicas:

* Obstrucción o ruptura de las barreras naturales.

* Mucoviscidosis, estenosis de vías urinarias, fugas de líquido cefalorraquídeo, dermatitis atópica o quemaduras.

- Causas inmunológicas:

* Pérdida de inmunoglobulinas por riñón o intestino.

* Infección por VIH.

* Tratamiento inmunosupresor.

* Neoplasias (leucemias, linfomas).
* Quimioterapia.

* Lupus eritematoso sistémico.

* Diabetes mellitus.

* Desnutrición.

Ante la sospecha clínica puede solicitarse estudios iniciales como los siguientes: $:^{1,3,8,20}$

1. Biometría hemática. La presencia de linfopenia orienta a una IDP de tipo celular (dependiente de linfocitos T). La neutropenia con $<500$ neutrófilos $/ \mathrm{mm}^{3}$ explica la ocurrencia de infecciones, sin embargo, un valor normal no descarta neutropenia cíclica. La citopenia autoinmune también se relaciona con IDP.

2. Inmunoglobulinas séricas (IgG, $\lg M, \lg A$, IgE). La IgG es valorable después de los 4 meses de vida extrauterina debido a que antes de esa edad es de origen materno. Es indispensable interpretar los niveles de acuerdo con los valores de referencia según la edad. La disminución de inmunoglogulinas sugiere inmunodeficiencia humoral (linfocitos B) o combinada (linfocitos T y B)

3. Pruebas de hipersensibilidad retardada (candidina). Con ellas se evalúa la inmunidad celular.

4. Ultrasonido o radiografía de tórax. En los lactantes sirve para evaluar la presencia de timo; se observa ausencia de timo en inmunodeficiencia combinada.

5. Complemento hemolítico total (CH50). Si los niveles son bajos es necesario solicitar cuantificación de C1-C9.

No obstante lo anterior, los resultados "normales" de las pruebas señaladas no descartan la existencia de IDP. Las pruebas que confirman el diagnóstico solo se realizan en centros especializados (en México, por ejemplo, la Unidad de Investigación en Inmunodeficiencias del Instituto Nacional de Pediatría es uno de ellos):3,14,20 
1. Identificación de poblaciones linfocitarias en sangre periférica. Se puede diferenciar entre los diferentes tipos de linfocitos mediante marcadores específicos como anticuerpos monoclonales fluorescentes, para ser detectados con un citómetro de flujo:

- Linfocitos B o CD19+, CD20+.

- Linfocitos T o CD3+, CD4+, CD8+.

- Linfocitos NK o CD16+/CD56+.

- Linfocitos B de memoria IgM-, IgD-, CD27+.

Los resultados de interpretan de acuerdo a la edad.

2. Respuesta a antígenos proteicos y polisacáridos (después de la aplicación de la vacuna contra tétanos y contra neumococo, respectivamente).

- Consiste en aplicar al paciente la vacuna polisacárida de neumococo (no conjugada) y enviar al día siguiente al laboratorio para la cuantificación de los anticuerpos contra 14 antígenos polisacáridos (que incluyan los de la vacuna aplicada). Un mes después es necesario medir nuevamente los niveles de anticuerpos contra antígenos polisacáridos (que incluyan los de la vacuna aplicada). Implica comparar los valores de anticuerpos antes y después de la vacuna:

* Para un niño mayor de 5 años debe existir un nivel superior a $1.3 \mathrm{mcg} / \mathrm{mL}$ en $70 \%$ de los anticuerpos estudiados.

* Para un niño menor de 5 años debe identificarse un valor mayor de 1.3 $\mathrm{mcg} / \mathrm{mL}$ en $50 \%$ de los anticuerpos estudiados.

* Cuando las cuantificaciones son menores a los rangos establecidos será necesario pensar en una deficiencia a antígenos polisacáridos.

3. Medición de subclases de inmunoglobulinas de $\lg G$, $\lg G 1$, $\lg G 2$, $\lg G 3$, $\lg G 4$. Se recomien- da ante niveles normales de $\lg G$ relacionados con infecciones recurrentes de vías respiratorias. Para que el resultado sea valorable se debe realizar después de los 18 meses de edad.

4. Detección de aglutininas (anti-A y anti-B) en grupos sanguíneos $\mathrm{A}$ o $\mathrm{B}$.

- Evalúa la producción de anticuerpos naturales contra antígenos polisacáridos.

- No puede realizarse antes de los 2 años de edad.

- No se debe solicitar en pacientes con grupo sanguíneo $A B$.

5. Linfoproliferación.

- Medición de la proliferación de los linfocitos con estímulos antigénicos no específicos (PHA, anticuerpos anti-CD3)

- Medición de la proliferación de los linfocitos con estímulos antigénicos como toxina antitetánica, candidina o tuberculina.

- Se solicita por sospecha de una IDP con déficit celular.

6. Evaluación de la producción de radicales libres por los neutrófilos (fagocitosis).

- Por técnica de 1,2,3 dihidrorodamina.

- Por técnica de nitroazul de tetrazolio.

La falta de producción de radicales libres confirmada por ambas técnicas es indicativa de enfermedad granulomatosa crónica.

En la Figura 1 se muestra el algoritmo para el diagnóstico de las IDP, en que están integrados los estudios descritos.

Otras pruebas para precisar el diagnóstico solo se realizan en laboratorios de los países desarrollados; los grupos médicos que lo requieran pueden comunicarse con los expertos que colaboran en ellos mediante el correo electrónico asentado en los artículos que dan a conocer el trabajo realizado en dichos centros. . $^{3,18,20}$ 


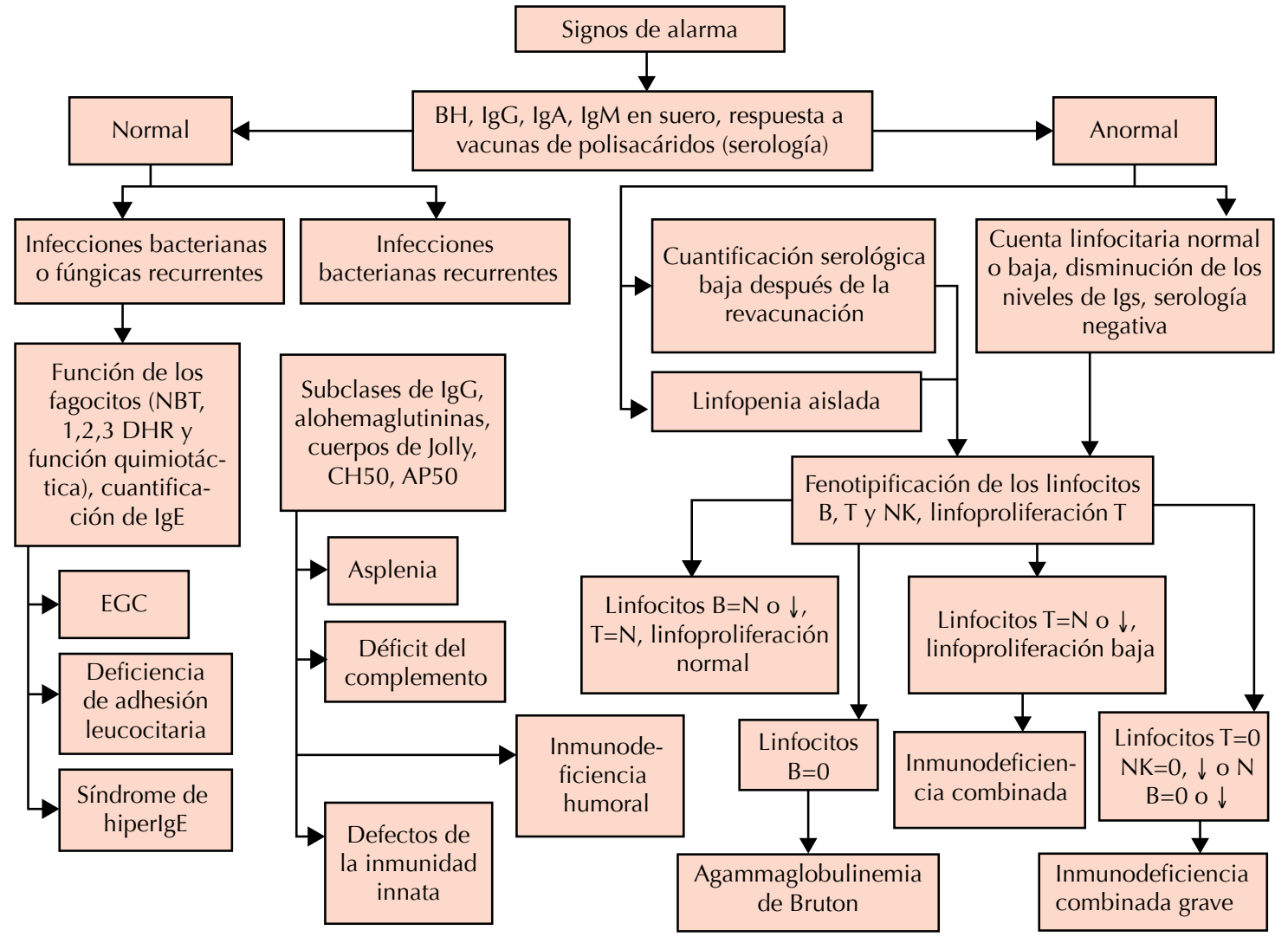

Figura 1. Algoritmo para diagnóstico de un paciente con probable IDP. BH: biometría hemática; IgG: inmunoglobulina G; IgA: inmunoglobulina A; IgM: inmunoglobulina M; NBT: nitroazul de tetrazolio; 1,2,3 DHR: dihidrorodamina; EGC: enfermedad granulomatosa crónica; Igs: inmunoglobulinas; $\mathrm{CH} 50$ : complemento hemolítico (vía clásica); AP50: vía alterna del complemento.

\section{TRATAMIENTO DE LAS INMUNODEFICIENCIAS}

De forma general, las infecciones en los pacientes con inmunodeficiencias requieren varios ciclos de antibióticos, esquemas más prolongados, dosis mayores o la administración de antibióticos intravenosos para una respuesta terapéutica favorable. Los antibióticos profilácticos se recomiendan en algunas IDP.

Otros tratamientos incluyen inmunomoduladores (interferón gamma recombinante), reemplazo de la inmunoglobulina recombinante intravenosa o subcutánea (la dosis óptima se determina con la ausencia de infecciones antes de la aplicación siguiente), terapia enzimática (deficiencia de adenosindeaminasa), trasplante de células progenitoras hematopoyéticas y terapia génica.

Otra medida que no debe ser olvidada por los médicos es el uso de productos radiados cuando sea necesaria una trasfusión sanguínea.

La fiebre, relacionada o no con infección, es un dato de alarma en los pacientes con IDP y 
requerirá un protocolo de estudio que incluya diversos cultivos y aislamiento en el hospital., ${ }^{3,21}$

En los pacientes con ciertas IDP deben evitarse las vacunas con agentes vivos atenuados, como la BCG, la Sabin oral (antipolio), aquellas contra rotavirus, fiebre amarilla, sarampión, parotiditis, rubéola y varicela. En general, se pueden aplicar las vacunas inactivadas, sin embargo, la inmunogenicidad es variable según el tipo de IDP. En ciertas IDP es recomendable la aplicación de vacunas como la antipneumococo, antihaemophilus influenzae tipo b y antimenigococo, como en los pacientes con asplenia o con déficit de complemento. Existen guías específicas de vacunación para cada IDP. ${ }^{17}$

Por otro lado, además de educar a los pacientes y a sus familiares en los aspectos específicos de las IDP, el personal médico no debería omitir formular las siguientes recomendaciones generales: ${ }^{18}$

1. La fiebre debe ser considerada un dato de alarma, tanto en los pacientes pediátricos como en los adultos.

2. Evitar la humedad en la casa y el uso de aire acondicionado (favorecen la inhalación esporas).

3. La limpieza periódica de los juguetes de los niños.

4. Evitar el tabaquismo, tanto el activo como el pasivo.

5. La ingestión de una dieta balanceada, así como evitar comer alimentos fuera de casa, alimentos crudos o poco cocidos (huevo, carne) o vegetales sin desinfectar (lechuga, espinacas, rábanos o cilantro, por ejemplo).

6. Evitar ingerir agua no potable.

7. Evitar inhalar café molido, té a granel, pimienta molida o sopas liofilizadas (pueden contener esporas de hongos).

8. Evitar asistir a lugares concurridos o donde pueda haber contacto con personas que cursen con alguna infección.
9. Evitar asistir a la escuela en caso de brotes epidémicos.

10. Evitar nadar en albercas, mar o ríos contaminados.

11. Evitar el contacto con mascotas (los lamidos, rasguños, mordeduras son vías de infección).

12. Procurar la adecuada higiene corporal y bucodental.

13. Limpieza continua de las manos.

14. Realizar lavados nasales con solución salina.

15. Lavar y curar las heridas.

16. Evitar las perforaciones corporales con finalidades cosméticas.

17. Drenar las secreciones con fisioterapia.

18. Consultar al médico especialista antes de aplicar una vacuna, incluso aquellas incluidas en las campañas del Sistema Nacional de Salud.

19. Evitar la exposición al polvo en los ambientes cotidianos en los que se desempeña el paciente.

20. Evitar la exposición a excretas de palomas.

\section{¿POR QUÉ NO SE DIAGNOSTICAN LAS INMUNODEFICIENCIAS PRIMARIAS?}

El retraso en el diagnóstico de las IDP es variable y depende del tipo de deficiencia; para algunas condiciones puede ser hasta de 10 años. El retraso se relaciona con mayor morbilidad. Las razones de la demora en el diagnóstico son diversas: ${ }^{3}$

1. Las infecciones recurrentes pueden ser aceptadas como variaciones de la normalidad.

2. Existe la percepción errónea de que las IDP se presentan exclusivamente durante la infancia.

3. Las características de las IDP pueden ser difíciles de distinguir de las condiciones de otras enfermedades, tales como las bronquiectasias, sarcoidosis, asma o enfermedad pulmonar obstructiva crónica. 
4. Las IDP pueden ser valoradas por médicos generales o especialistas como pediatras, internistas, gastroenterólogos, hematólogos u reumatólogos, quienes no están familiarizados con este grupo de enfermedades

\section{AGRADECIMIENTOS}

Agradecemos a la Fundación Mexicana de Niñas y Niños con Inmunodeficiencias Primarias A.C. (FUMENI), así como a la doctora Selma Scheffler, por la revisión y sugerencias realizadas a este artículo.

\section{REFERENCIAS}

1. Suárez F. Immune deficit. Rev Prat. 2010;60(4):551-558.

2. Conle ME, Notarangelo LD, Casanova JL. Definition of primary immunodeficiency in 2011: a "trialogue" among friends. Ann N Y Acad Sci. 2011;1238:1-6.

3. Chapel H, Prevot J, Gaspar HB, Español T, Bonilla FA, Solis L, et al. Primary immune deficiencies. Principles of care. Front Immunol. Front Immunol. 2014 Dec 15;5:627.

4. Group CTFPs. The French National Registry Of Primary Immunodeficiency Diseases. Clin Immunol. 2010;135(2):264-272.

5. Condino-Neto A. The relevance of collaborative work: the Latin American Society for Immunodeficiencies (LASID) registry model. Clin Exp Immunol. 2014;178 Suppl 1: 16-17.

6. Abel L, EL Baghdadi J, Bousfiha AA, Casanova JL, Schurr E. Human genetics of tuberculosis: a long and winding road. Philos Trans R Soc Lond B Biol Sci. 2014;369(1645):20130428.

7. Picard C, Al-Herz W, Bousfiha A, Casanova JL, Chatila T, Conley ME, et al. Primary immunodeficiency diseases: an update on the Classification from the International Union of Immunological Societies Expert Committee for Primary Immunodeficiency 2015. J Clin Immunol. 2015;35(8):696-726.

8. Routes J, Abinun M, Al-Herz W, Bustamante J, CondinoNeto A, De La Morena MT, et al. ICON: the early diagnosis of congenital immunodeficiencies. J Clin Immunol. 2014;34(4):398-424.

9. Bousfiha AA, Jeddane L, El Hafidi N, Benajiba N, Rada N, El Bakkouri J, et al. First report on the Moroccan registry of primary immunodeficiencies: 15 years of experience (1998-2012). J Clin Immunol. 2014;34(4):459-468.
10. Aghamohammadi A, Mohammadinejad $\mathrm{P}$, Abolhassani $\mathrm{H}$, Mirminachi B, Movahedi M, Gharagozlou M, et al. Primary immunodeficiency disorders in Iran: update and new insights from the third report of the national registry. J Clin Immunol. 2014;34(4):478-490.

11. Marschall $K$, Hoernes $M$, Bitzenhofer-Grüber $M$, Jandus $P$, Duppenthaler A, Wuillemin WA, et al, The Swiss National Registry for Primary Immunodeficiencies: report on the first 6 years' activity from 2008 to 2014. Clin Exp Immunol. 2015;182(1):45-50.

12. European Society for Immunodeficiencies [sitio web]. Welcome to the Registry Working Party! Disponible en http://esid.org/Working-Parties/Registry.

13. Wu UI, Holland SM. Host susceptibility to non-tuberculous mycobacterial infections. Lancet Infect Dis. 2015;15(8):968980.

14. Bousfiha AA, Jeddane L, Ailal F, Al Herz W, Conley ME, Cunningham-Rundles $C$, et al. A phenotypic approach for IUIS PID classification and diagnosis: guidelines for clinicians at the bedside. J Clin Immunol. 2013;33(6):107887.

15. Casanova JL. Human genetic basis of interindividual variability in the course of infection. Proc Natl Acad Sci U S A. 2015;112(51):E7118-E7127.

16. Lanternier F, Mahdaviani SA, Barbati E, Chaussade H, Koumar Y, Levy R, et al. Inherited CARD9 deficiency in otherwise healthy children and adults with Candida species-induced meningoencephalitis, colitis, or both. J Allergy Clin Immunol. 2015;135(6):1558-68 e2.

17. Bezrodnik L, Di Giovanni D, Gómez Raccio A, Paz R, Regairaz $L$, et al. Guías de manejo: vacunas en pacientes con inmunodeficiencias primarias. Arch Argent Pediatr. 2010;108(5):454-464.

18. CEREDIH, Le Centre de Référence Déficits Immunitaires Héréditaires [sitio web]. Home. Disponible en http:// www.ceredih.fr/

19. Lugo Reyes SO, Ramirez-Vazquez G, Cruz Hernández A, Medina-Torres EA, Ramirez-Lopez AB, España-Cabrera C, et al. Clinical features, non-infectious manifestations and survival analysis of 161 children with primary immunodeficiency in Mexico: a single center experience over two decades. J Clin Immunol. 2016 Jan;36(1):56-65.

20. Picard $\mathrm{C}$. [How to diagnose a hereditary immunodeficiency?]. Rev Prat. 2007;57(15):1671-166.

21. Aguilar C, Malphettes M, Donadieu J, Chandesris $O$, Coignard-Biehler $\mathrm{H}$, Catherinot $\mathrm{E}$, et al. Prevention of infections during primary immunodeficiency. Clin Infect Dis. 2014;59(10):1462-1470. 